VOL. 52 (1995) [359-372]

\title{
TRACE FUNCTIONS ON INVERSE SEMIGROUP ALGEBRAS
}

\author{
D. Easdown AND W.D. MUNN
}

Let $S$ be an inverse semigroup and let $F$ be a subring of the complex field containing 1 and closed under complex conjugation. This paper concerns the existence of trace functions on $F[S]$, the semigroup algebra of $S$ over $F$. Necessary and sufficient conditions on $S$ are found for the existence of a trace function on $F[S]$ that takes positive integral values on the idempotents of $S$. Although $F[S]$ does not always admit a trace function, a weaker form of linear functional is shown to exist for all choices of $S$. This is used to show that the natural involution on $F[S]$ is special. It also leads to the construction of a trace function on $F[S]$ for the case in which $F$ is the real or complex field and $S$ is completely semisimple of a type that includes countable free inverse semigroups.

The concept of a trace function on a real or complex algebra had its origin in matrix theory and is of central importance in many algebraic and analytical contexts. In the case of a group algebra, the trace of an element is defined simply to be the coefficient of the identity and is easily seen to possess all the standard properties. With the growth of interest in inverse semigroups (a class of involution semigroups with many group-like features), it is natural to ask whether the corresponding semigroup algebras also admit trace functions.

In this paper we consider the semigroup algebra $F[S]$ of an inverse semigroup $S$ over a subring $F$ of $\mathbb{C}$ that contains 1 and is closed under complex conjugation. In Section 1, where the basic definitions appear, two simple necessary conditions are obtained for the existence of a trace function on $F[S]$ and attention is drawn to those trace functions (called 'strong') with the property that their values on the idempotents of $S$ are positive integers. The main result of Section 2 provides a necessary and sufficient condition for $F[S]$ to admit a strong trace function - namely that each principal ideal of the semilattice of $S$ be finite. Section 3 comprises two examples.

The notion of a pseudotrace function relative to a submodule is introduced in Section 4 and it is shown that, for any nonempty finite subset $T$ of $S, F[S]$ admits a

Received 17 January 1995

The authors wish to record their thanks to Dr. M.J. Crabb, University of Glasgow, for many helpful suggestions.

Copyright Clearance Centre, Inc. Serial-fee code: 0004-9729/95 \$A2.00+0.00. 
pseudotrace function relative to $F[T]$. This result is applied in Section 5 to show that the natural involution on $F[S]$ is 'special', in the sense of the authors' definition in [4].

Finally, in Section 6, pseudotraces are used to construct a trace function on the real or complex semigroup algebra of an inverse semigroup in which each $\mathcal{D}$-class contains finitely many idempotents and the set of all $\mathcal{D}$-classes is countably infinite. Semigroups of this type include free inverse semigroups of finite or countably infinite rank.

\section{Preliminaries}

The notation and terminology for semigroups will be that of [2]. As is customary, the cardinal of a set $X$ will be denoted by $|X|$.

Throughout, $F$ denotes a subring of $\mathbb{C}$ that contains 1 and is closed under complex conjugation (written $\alpha \mapsto \bar{\alpha}$ ). We write

$$
F^{+}:=\{\alpha \in F: \alpha=\bar{\alpha}>0\}, \quad F^{+0}:=F^{+} \cup\{0\} .
$$

In particular, $\mathbb{N}\left(=\mathbb{Z}^{+}\right) \subseteq F^{+}$.

Let $A$ be an $F$-algebra that admits an involution * (a mapping *: $A \rightarrow A$ such that, for all $a, b \in A$ and all $\lambda \in F,(a+b)^{*}=a^{*}+b^{*},(a b)^{*}=b^{*} a^{*}, a^{* *}=a$, $(\lambda a)^{*}=\bar{\lambda} a^{*}$.) By a trace function on $A$ with respect to ${ }^{*}$ we mean a mapping $\chi: A \rightarrow$ $F$ satisfying the following five conditions:

T1. $\chi$ is $F$-linear.

T2. $(\forall a, b \in A) \chi(a b)=\chi(b a)$.

T3. $(\forall a \in A) \chi\left(a^{*}\right)=\overline{\chi(a)}$.

T4. $(\forall a \in A) \chi\left(a a^{*}\right) \in F^{+0}$.

T5. $(\forall a \in A) \chi\left(a a^{*}\right)=0 \Rightarrow a=0$.

The set of idempotents of $A$ is partially ordered by the relation $\geqslant$ defined by the rule that, for idempotents $a$ and $b, a \geqslant b$ if and only if $a b=b a=b$. We call $a \in A$ a projection if and only if $a^{2}=a=a^{*}$. The following simple observation will be used frequently.

1.1. Let $\chi$ be a trace function on $A$ and let $a, b, c$ be projections in $A$.

(i) If $a \neq 0$ then $\chi(a) \in F^{+}$.

(ii) If $b>c$ then $\chi(b)>\chi(c)$.

Result (i) is an immediate consequence of $\mathrm{T} 4$ and $\mathrm{T} 5$. For (ii), we note that if $b>c$ then $b-c$ is a nonzero projection and so, by (i), $\chi(b-c) \in F^{+}$; that is, by T1, $\chi(b)-\chi(c) \in F^{+}$.

Now let $S$ be an inverse semigroup and let $F[S]$ denote the semigroup algebra of $S$ over $F$ (see, for example, [6, Section 1]). A typical element $a \in F[S]$ can be written 
in the form $\sum_{x \in S} \alpha_{x} x\left(\alpha_{x} \in F\right)$ and we write $\operatorname{supp}(a):=\left\{x \in S: \alpha_{x} \neq 0\right\}$ (a finite subset of $S$, which is empty if and only if $a=0$ ). It is easily verified that the mapping *:F[S] $\rightarrow F[S]$ defined by

$$
\left(\sum_{x \in S} \alpha_{x} x\right)^{*}=\sum_{x \in S} \bar{\alpha}_{x} x^{-1} \quad\left(\alpha_{x} \in F\right)
$$

is an involution on $F[S]$. We call this the natural involution on $F[S]$. Let $E$ denote the semilattice of $S$, that is, the subsemigroup $\left\{e \in S: e^{2}=e\right\}$. Clearly $F[E]$ is a commutative subalgebra of $F[S]$. Note also that every element of $E$ is a projection in $F[S]$.

Our aim is to examine trace functions on $F[S]$. It will be assumed throughout that these are with respect to the natural involution on $F[S]$. That such functions need not always exist is clear from the proposition below. Recall that $S$ is completely semisimple if and only if each of its principal factors is either a Brandt semigroup [2, Section 3.3] or (in the case of the least ideal of $S$, where this exists) a group.

Proposition 1.2. Let $F, S$ and $E$ be as above and suppose that $F[S]$ admits a trace function. Then

(i) $S$ is completely semisimple,

(ii) every totally ordered subset of $E$ has cardinal not exceeding $\left|F^{+}\right|$.

Proof: Let $\chi$ be a trace function on $F[S]$.

(i) Suppose that $S$ is not completely semisimple. Then $S$ contains a subsemigroup $B$ isomorphic to the bicyclic semigroup [2, Theorem 2.54]. Thus there exist $e, f \in E$ and $p, q \in S$ such that $p q=e>f=q p$. Since $e$ and $f$ are projections in $F[S]$ it follows from 1.1(ii) that $\chi(e)>\chi(f)$ in $F^{+}$. Hence $\chi(p q)>\chi(q p)$, which contradicts T2.

(ii) Let $C$ be a totally ordered subset of $E$ (with respect to the natural ordering) and let $e, f$ be distinct elements of $C$. Then either $e>f$ or $f>e$ and so, by 1.1(ii), $\chi(e) \neq \chi(f)$. Hence $\left.\chi\right|_{C}: C \rightarrow F^{+}$is injective, which shows that $|C| \leqslant\left|F^{+}\right|$.

On the other hand, if $S$ is a group, with identity 1 , then, as is well known, the mapping $\chi: F[S] \rightarrow F$ defined by $\chi\left(\sum_{x \in S} \alpha_{x} x\right)=\alpha_{1}$ is a trace function on $F[S]$. Another case in which a trace function always exists is that in which $S$ is finite: for in this case it can be shown that the Vagner-Preston representation of $S$ by partial $1-1$ transformations of $S$ [2, Theorem 1.20] leads to a faithful matrix representation of $F[S$ ] (see Section 2) and the usual matrix trace function on the image algebra induces a trace function $\chi$ on $F[S]$ itself. 
In each of these cases $\chi$ satisfies the further condition:

T6. $(\forall e \in E) \quad \chi(e) \in \mathbb{N}$.

(The zero of $S$, if such an element exists, is not identified with the zero of $F[S]$.) A trace function on $F[S]$ satisfying $\mathrm{T} 6$ will be termed strong. Note that if $F=\mathbb{Z}$ then $F^{+}=\mathbb{N}$ and so, by $1.1(\mathrm{i})$, every trace function on $F[S]$ is strong.

An example of an inverse semigroup whose algebra over $\mathbb{Q}$ admits a trace function but no strong trace function is provided in Section 3.

\section{Strong trace FunCtions}

In this section we obtain necessary and sufficient conditions on an inverse semigroup $S$ for $F[S]$ to admit a strong trace function.

We begin by defining a mapping ( | $: F[S] \times F[S] \rightarrow F$ by

$$
\left(\sum_{x} \alpha_{x} x \mid \sum_{x} \beta_{x} x\right):=\sum_{x} \alpha_{x} \bar{\beta}_{x} \quad\left(\alpha_{x}, \beta_{x} \in F\right)
$$

The following result is well known and easily verified.

LEMMA 2.1. ( $\mid$ ) is a hermitian inner product on $F[S]$; that is, for all $a, b, c \in$ $F[S]$ and all $\lambda \in F$,

(i) $(a+b \mid c)=(a \mid c)+(b \mid c)$,

(ii) $(\lambda a \mid b)=\lambda(a \mid b)$,

(iii) $(a \mid b)=\overline{(b \mid a)}$,

(iv) $(a \mid a) \in F^{+0}$,

(v) $(a \mid a)=0 \Rightarrow a=0$.

We shall also make use of the representation (described below) of $F[S]$ by linear transformations of $F[S]$ induced by the Vagner-Preston representation of $S$ by one-toone partial transformations of $S$ [2, Theorem 1.20]. This was developed by Barnes [1] into a representation of $\mathbb{C}[S]$ by bounded linear operators on a Hilbert space.

For $a=\sum_{y \in S} \alpha_{y} y \in F[S]$ we define a linear transformation $\theta_{a}$ of $F[S]$ by the rule that, on the basis $S, \theta_{a}$ is given by

$$
x \theta_{a}:=\sum_{\substack{y \in S \\ x y y^{-1}=x}} \alpha_{y}(x y) \quad(x \in S)
$$

We then define a mapping $\theta$ from $F[S]$ into the $F$-algebra of linear transformations of $F[S]$ by taking $a \theta:=\theta_{a}(a \in F[S])$. Clearly $\theta$ is $F$-linear. 
Lemma 2.2. For all $a, b, c \in F[S]$ and all $x \in S$,

(i) $\theta_{a} \theta_{b}=\theta_{a b}$,

(ii) $\left(a \theta_{b} \mid c\right)=\left(a \mid c \theta_{b^{*}}\right)$,

(iii) $x \theta_{a}=x\left(\left(x^{-1} x\right) \theta_{a}\right)$,

(iv) $a^{*} \theta_{a}=0 \Rightarrow a=0$.

Proof: Parts (i) and (ii), which together assert that $\theta$ is a 'star-representation' of $F[S]$, are essentially established in [1, Proposition 2.1].

(iii) Let $x, y \in S$. Then

$$
x\left(\left(x^{-1} x\right) \theta_{y}\right)= \begin{cases}x y & \text { if } x^{-1} x y y^{-1}=x^{-1} x \\ 0 & \text { otherwise. }\end{cases}
$$

But $x^{-1} x y y^{-1}=x^{-1} x$ if and only if $x y y^{-1}=x$. Hence $x\left(\left(x^{-1} x\right) \theta_{y}\right)=x \theta_{y}$. Thus, by linearity, $x\left(\left(x^{-1} x\right) \theta_{a}\right)=x \theta_{a}$ for all $x \in S$ and all $a \in F[S]$.

(iv) Let $a=\sum_{x \in S} \alpha_{x} x \in F[S] \backslash\{0\}$. Then

$$
a^{*} \theta_{a}=\sum_{x, y} \overline{\alpha_{x}} \alpha_{y}\left(x^{-1} \theta_{y}\right)=\sum_{\substack{x, y \\ x^{-1} y y^{-1}=x^{-1}}} \overline{\alpha_{x}} \alpha_{y}\left(x^{-1} y\right) .
$$

Now, by [4, Theorem 1(i)], there exists $t \in \operatorname{supp}(a)$ such that, for all $x, y \in \operatorname{supp}(a)$, $t^{-1} t=x^{-1} y$ implies $x=y$. Thus the coefficient of $t^{-1} t$ in $a^{*} \theta_{a}$ is $\sum\left|\alpha_{x}\right|^{2}$, where the summation is over all $x \in S$ with $x^{-1} x=t^{-1} t$, and this is nonzero. Hence $a^{*} \theta_{a} \neq 0$.

REMARK. By (iv), the representation $\theta$ of $F[S]$ is faithful.

We now come to the main result of this section .

THEOREM 2.3. Let $S$ be an inverse semigroup with semilattice $E$ and let $F$ be a subring of $\mathbb{C}$ containing 1 and closed under complex conjugation. Then $F[S]$ admits a strong trace function if and only if each principal ideal of $E$ is finite.

Proof: Suppose that $F[S]$ admits a strong trace function $\chi$. Let $z \in E$ be such that $\chi(z)$ is minimal in $\{\chi(e): e \in E\}$ (a subset of $\mathbb{N}$ ). Then $z$ is the zero of $E$ : for otherwise there exists $f \in E$ such that $z>z f$ and so, by 1.1(ii), $\chi(z)>\chi(z f)$, contrary to the choice of $z$. Hence $|E z|=1$.

We proceed by induction. Let $e \in E$, where $e \neq z$, let $k=\chi(e)(\in \mathbb{N})$ and assume that $|E f|<\infty$ for all $f \in E$ such that $\chi(f)<k$. First, let $e_{1}, e_{2}, \ldots, e_{m} \in E$ be such that $e \geqslant e_{1}>e_{2}>\ldots>e_{m}$. Then $k \geqslant \chi\left(e_{1}\right)>\chi\left(e_{2}\right)>\ldots>\chi\left(e_{m}\right)$, by 1.1(ii). Hence, since $\chi\left(e_{i}\right) \in \mathbb{N}(i=1, \ldots, m)$, we have that $m \leqslant k$. It follows that

$$
(\forall g \in E \text { with } e>g)(\exists f \in E) e \succ f \geqslant g,
$$


where we write $e \succ f$ to mean that $e>f$ and there is no $h \in E$ with $e>h>f$. Write $C:=\{f \in E: e \succ f\}$. From 2.3a, $C \neq \emptyset$. Suppose that $f_{1}, f_{2}, \ldots, f_{n}$ are distinct elements of $C$. We define $u_{1}, u_{2}, \ldots, u_{n} \in F[S]$ by

$$
u_{i}:=\left(e-f_{1}\right)\left(e-f_{2}\right) \ldots\left(e-f_{i}\right) \quad(i=1,2, \ldots, n) .
$$

Then each $u_{i}$ is a projection in $F[S]$ and $e>u_{1} \geqslant u_{2} \geqslant \ldots \geqslant u_{n}>0$. Suppose that $u_{i}=u_{i+1}$ for some $i \in\{1,2, \ldots, n-1\}$. Thus

$$
u_{i}=u_{i}\left(e-f_{i+1}\right)=u_{i}-u_{i} f_{i+1}
$$

and so $u_{i} f_{i+1}=0$. Now $u_{i}=e-a$, where

$$
a \in F[E] f_{1}+F[E] f_{2}+\ldots+F[E] f_{i}
$$

and therefore $0=e f_{i+1}-a f_{i+1}=f_{i+1}-a f_{i+1}$. But, for all $j \in\{1, \ldots, i\}, f_{i+1} \notin E f_{j}$ (since $e \succ f_{i+1}$ ) and so $f_{i+1} \neq a f_{i+1}$, contrary to our previous conclusion. Hence $e>u_{1}>u_{2}>\ldots>u_{n}>0$. Consequently, by $1.1($ ii),

$$
k=\chi(e)>\chi\left(u_{1}\right)>\chi\left(u_{2}\right)>\ldots>\chi\left(u_{n}\right)>0 .
$$

But $\chi\left(u_{i}\right) \in \mathbb{Z}$, since $u_{i} \in \mathbb{Z}[E]$ and $\chi(g) \in \mathbb{N}$ for each $g \in E(i=1, \ldots, n)$. Hence, from $2.3 \mathrm{~b}, n<k$. Thus $|C|<k$.

Now let the elements of $C$ be $f_{1}, f_{2}, \ldots, f_{r}$ (where $r<k$ ). Then, from 2.3a, $E e=\{e\} \cup\left(\bigcup_{i=1}^{r} E f_{i}\right)$ and so

$$
|E e| \leqslant 1+\sum_{i=1}^{r}\left|E f_{i}\right| .
$$

But, by 1.1(ii), $\chi\left(f_{i}\right)<\chi(e)=k$ and so, by the inductive hypothesis, $\left|E f_{i}\right|<\infty$ $(i=1,2, \ldots, r)$. Thus $|E e|<\infty$. It follows by induction that $|E e|<\infty$ for all $e \in E$.

Conversely, suppose that $|E f|<\infty$ for all $f \in E$. For $x \in S$ and $e \in E$, $\left(e \theta_{x} \mid e\right) \neq 0$ implies $e \leqslant x x^{-1}$. Hence, for all $x \in S,\left|\left\{e \in E:\left(e \theta_{x} \mid e\right) \neq 0\right\}\right|<\infty$. With this in mind, we define $\chi: F[S] \rightarrow F$ by

$$
\chi(a):=\sum_{e \in E}\left(e \theta_{a} \mid e\right) \quad(a \in F[S])
$$

with the convention that we regard the sum as finite. We show that $\chi$ is a strong trace function on $F[S]$ : 
The linearity of $\chi$ (T1) follows from 2.1(i), (ii) and the linearity of $\theta$. We now verify that $\mathrm{T} 2, \ldots, \mathrm{T} 6$ hold.

First, we note that if $e \in E$ and $x \in S$ are such that $e x=e$ then $e=e x(e x)^{-1}=$ $e x x^{-1}$; hence $\left(e \theta_{x} \mid e\right)=1$ if $e x=e$ and is zero otherwise. It follows that

$$
(\forall x \in S) \quad \chi(x)=|\{e \in E: e x=e\}| .
$$

Now let $x, y \in S$. We show that $\chi(x y)=\chi(y x)$. Define nonempty finite subsets $A$ and $B$ of $E$ by

$$
A:=\{e \in E: e x y=e\}, \quad B:=\{f \in E: f y x=f\} .
$$

For all $e \in A$ we have that $\left(x^{-1} e x\right) y x=x^{-1}(e x y) x=x^{-1} e x \in E$ and so $x^{-1} e x \in B$. We may therefore define $\varphi: A \rightarrow B$ by $e \varphi=x^{-1} e x(e \in A)$. Suppose that $e, e^{\prime} \in A$ are such that $e \varphi=e^{\prime} \varphi$. Then

$$
e=e x y=x x^{-1} e x y=x(e \varphi) y=x\left(e^{\prime} \varphi\right) y=x x^{-1} e^{\prime} x y=e^{\prime} x y=e^{\prime} .
$$

Thus $\varphi$ is injective and so $|A| \leqslant|B|$. Similarly, it can be shown that $|B| \leqslant|A|$. Hence, from 2.3c, $\chi(x y)=|A|=|B|=\chi(y x)$. Consequently, for all $a, b \in F[S]$, $\chi(a b)=\chi(b a)$; that is, T2 holds.

Next, let $a \in F[S]$. Then, from 2.2(ii) and 2.1(iii),

$$
\chi\left(a^{*}\right)=\sum_{e \in E}\left(e \theta_{a^{*}} \mid e\right)=\sum_{e \in E}\left(e \mid e \theta_{a}\right)=\sum_{e \in E} \overline{\left(e \theta_{a} \mid e\right)}=\overline{\chi(a)} ;
$$

that is; T3 holds. Also, from 2.2(i), (ii) and 2.1(iv),

$$
\chi\left(a a^{*}\right)=\sum_{e \in E}\left(e \theta_{a a^{*}} \mid e\right)=\sum_{e \in E}\left(e \theta_{a} \theta_{a^{*}} \mid e\right)=\sum_{e \in E}\left(e \theta_{a} \mid e \theta_{a}\right) \in F^{+0},
$$

which establishes T4. Now suppose that $a \neq 0$. By 2.2(iv), $a^{*} \theta_{a} \neq 0$ and so there exists $x \in \operatorname{supp}\left(a^{*}\right)$ such that $x \theta_{a} \neq 0$. Hence, by $2.2(i i i),\left(x^{-1} x\right) \theta_{a} \neq 0$. Thus, by $2.1(v)$,

$$
\chi\left(a a^{*}\right)=\sum_{e \in E}\left(e \theta_{a} \mid e \theta_{a}\right) \geqslant\left(\left(x^{-1} x\right) \theta_{a} \mid\left(x^{-1} x\right) \theta_{a}\right)>0
$$

and so T5 holds. Finally, we note that T6 holds; for if $f \in E$ then, by $2.3 c, \chi(f)=$ $|E f| \in \mathbb{N}$. Thus $\chi$ is a strong trace function on $F[S]$.

REMARK. It was shown in [6, Theorem 6.5] that if every principal ideal of $E$ is finite then $F[S]$ is a direct sum of ideals, indexed by the $\mathcal{D}$-classes of $S$, each ideal being isomorphic to a matrix ring (of possibly infinite type) over an appropriate group algebra. 


\section{TWO EXAMPLES}

We first give an example of an inverse semigroup algebra over $\mathbb{Q}$ which admits a trace function, but no strong trace function.

EXAMPLE 3.1. Let $E$ denote the semilattice (commutative semigroup of idempotents) $\left\{e_{i}: i \in \mathbb{N}\right\}$, where $e_{1}>e_{2}>e_{3}>\ldots$, let $\theta: E \rightarrow \mathbb{Q}^{+}$be a strictly order-preserving mapping and let $\chi: \mathbb{Q}[E] \rightarrow \mathbb{Q}$ be defined by extending $\theta$ by linearity. Clearly $\chi$ satisfies $\mathrm{T} 1, \mathrm{~T} 2$ and $\mathrm{T} 3$.

A typical element $a \in \mathbb{Q}[E]$ may be written in the form $\sum_{i=1}^{n} \alpha_{i} e_{i}$, where $\alpha_{i} \in \mathbb{Q}$ $(i=1,2, \ldots, n)$. Write $w_{i}:=e_{i}-e_{i+1}(i=1,2, \ldots, n-1)$ and $w_{n}:=e_{n}$. Then

$$
a=\beta_{1} w_{1}+\beta_{2} w_{2}+\ldots+\beta_{n} w_{n}
$$

where $\beta_{i}=\sum_{j=1}^{i} \alpha_{j}$. Now each $w_{i}$ is a nonzero projection in $\mathbb{Q}[E]$ and $w_{i} w_{j}=0$ if $i \neq j$. Hence

$$
a a^{*}=\beta_{1}^{2} w_{1}+\beta_{2}^{2} w_{2}+\ldots+\beta_{n}^{2} w_{n}
$$

and so

$$
\chi\left(a a^{*}\right)=\beta_{1}^{2} \chi\left(w_{1}\right)+\beta_{2}^{2} \chi\left(w_{2}\right)+\ldots+\beta_{n}^{2} \chi\left(w_{n}\right) .
$$

But $\chi\left(w_{n}\right) \in \mathbb{Q}^{+}$and, since $\theta$ is strictly order-preserving, $\chi\left(w_{i}\right) \in \mathbb{Q}^{+}$for $i=$ $1,2, \ldots, n-1$. Hence $\chi\left(a a^{*}\right) \in \mathbb{Q}^{+0}$; that is, T4 holds. Moreover, if $\chi\left(a a^{*}\right)=0$ then $\beta_{1}=\beta_{2}=\ldots=\beta_{n}=0$ and so $\alpha_{1}=\alpha_{2}=\ldots=\alpha_{n}=0$; that is, $a=0$. Thus T5 holds. Consequently, $\chi$ is a trace function on $\mathbb{Q}[E]$. However, since $E\left(=E e_{1}\right)$ is infinite, it follows that $\mathbb{Q}[E]$ does not admit a strong trace function.

In passing, we remark that the construction above shows that $\mathbb{Q}[E]$ admits $2^{\aleph_{0}}$ essentially distinct trace functions (in the sense that none is a nonzero scalar multiple of another.)

The second example is of an inverse semigroup which satisfies the necessary conditions given in 1.2 for the existence of a trace function on its semigroup algebra over $F$, but which admits no such function.

EXAMPLE 3.2. Let $S=(\mathbb{N} \times \mathbb{N}) \cup\{e, z\}$ and define a multiplication in $S$ by the following rules:

$$
\begin{gathered}
(i, j)(k, \ell)= \begin{cases}(i, \ell) & \text { if } j=k, \\
z & \text { otherwise, }\end{cases} \\
e(i, j)=(i, j) e=(i, j), \quad e^{2}=e, \\
z(i, j)=(i, j) z=z^{2}=z, \quad e z=z e=z .
\end{gathered}
$$


Thus $S$ is the inverse monoid obtained by adjoining an identity to the semigroup of $\mathbb{N} \times \mathbb{N}$ matrix units. The semilattice $E$ of $S$ is

$$
\{(i, i): i \in \mathbb{N}\} \cup\{e, z\} .
$$

It is easy to see that $S$ is completely semisimple and that a totally ordered subset of $E$ can have at most three elements.

Suppose that $F[S]$ admits a trace function $\chi$. Let $\chi(e)=\alpha$ and $\chi((1,1)-z)=\beta$. Since $e$ and $(1,1)-z$ are nonzero projections in $F[S]$ with $e>(1,1)-z$, it follows from 1.1 that $\alpha, \beta \in F^{+}$and $\alpha>\beta$. Choose $n \in \mathbb{N}$ such that $n \beta \geqslant \alpha$ and write

$$
p:=(1,1)+(2,2)+\ldots+(n, n)-n z .
$$

Since $p$ is the sum of the nonzero pairwise-orthogonal projections $(i, i)-z$ $(i=1,2, \ldots, n), p$ is a nonzero projection in $F[S] ;$ also $e>p$. Hence, by 1.1, $\chi(p) \in F^{+}$and $\alpha>\chi(p)$. But, for all $i \in\{1,2, \ldots, n\}$,

$$
\begin{aligned}
\chi((i, i)-z) & =\chi((i, 1)(1, i))-\chi(z)=\chi((1, i)(i, 1))-\chi(z) \\
& =\chi((1,1)-z)=\beta,
\end{aligned}
$$

by T1 and T2. Hence $\chi(p)=n \beta$ and so $\chi(p) \geqslant \alpha$, contradicting our previous conclusion. Thus $F[S]$ admits no trace function.

\section{Pseudotrace functions}

Let $A$ be an $F$-algebra that admits an involution * and let $B$ be a submodule of $A$. By a pseudotrace function on $A$ relative to $B$ (and with respect to ${ }^{*}$ ) we mean a mapping $\chi: A \rightarrow F$ satisfying the four conditions below:

T1. $\chi$ is $F$-linear.

T3. $(\forall a \in A) \chi\left(a^{*}\right)=\overline{\chi(a)}$.

T4. $(\forall a \in A) \chi\left(a a^{*}\right) \in F^{+0}$.

T5'. $(\forall a \in B) \chi\left(a a^{*}\right)=0 \Rightarrow a=0$.

Such functions on $F[S]$, where $S$ is an inverse semigroup, are assumed throughout to be with respect to the natural involution. We show that if $S$ is an arbitrary inverse semigroup then, for any nonempty finite subset $T$ of $S, F[S]$ admits a pseudotrace function relative to the submodule $F[T]$. Applications of this result are given in the next two sections.

With the notation of Section 2, for a nonempty finite subset $T$ of an inverse semigroup $S$, we define $\chi^{T}: F[S] \rightarrow F$ by

$$
\chi^{T}(a):=\sum_{t \in T}\left(t^{-1} \theta_{a} \mid t^{-1}\right) \quad(a \in F[S])
$$


Theorem 4.1. Let $S$ be an inverse semigroup, let $T$ be a nonempty finite subset of $S$ and let $F$ be a subring of $\mathbb{C}$ containing 1 and closed under complex conjugation. Then $\chi^{T}$ is a pseudotrace function on $F[S]$ relative to $F[T]$.

Proof: The linearity of $\chi^{T}$ follows from that of $\theta$; thus T1 holds. Let $a \in F[S]$. Then

$$
\chi^{T}\left(a^{*}\right)=\sum_{t \in T}\left(t^{-1} \theta_{a^{*}} \mid t^{-1}\right)=\sum_{t \in T}\left(t^{-1} \mid t^{-1} \theta_{a}\right)=\sum_{t \in T} \overline{\left(t^{-1} \theta_{a} \mid t^{-1}\right)}=\overline{\chi^{T}(a)}
$$

and

$$
\chi^{T}\left(a a^{*}\right)=\sum_{t \in T}\left(t^{-1} \theta_{a a^{*}} \mid t^{-1}\right)=\sum_{t \in T}\left(t^{-1} \theta_{a} \theta_{a^{*}} \mid t^{-1}\right)=\sum_{t \in T}\left(t^{-1} \theta_{a} \mid t^{-1} \theta_{a}\right) \in F^{+0}
$$

that is, T3 and T4 hold. Finally, suppose that $a \in F[T] \backslash\{0\}$. Then by 2.2(iv), $a^{*} \theta_{a} \neq 0$ and so there exists $u \in \operatorname{supp}\left(a^{*}\right)$ such that $u \theta_{a} \neq 0$. But $\operatorname{supp}\left(a^{*}\right) \subseteq T^{-1}$. Hence

$$
\chi^{T}\left(a a^{*}\right)=\sum_{t \in T}\left(t^{-1} \theta_{a} \mid t^{-1} \theta_{a}\right) \geqslant\left(u \theta_{a} \mid u \theta_{a}\right)>0,
$$

and so T5' holds.

Note. If $a=\sum_{x \in S} \alpha_{x} x \in F[S]$ then

$$
\chi^{T}(a)=\sum_{t \in T} \sum_{x \in S} \alpha_{x}\left(t^{-1} \theta_{x} \mid t^{-1}\right)=\sum_{t \in T} \sum_{\substack{x \in S \\ t^{-1} x=t^{-1}}} \alpha_{x}
$$

for $\left(t^{-1} \theta_{x} \mid t^{-1}\right)=1$ if $t^{-1} x=t^{-1}$ and is zero otherwise. (Observe that if $t^{-1} x=t^{-1}$ then $t t^{-1}=t t^{-1} x=t t^{-1} x\left(t t^{-1} x\right)^{-1}=t t^{-1} x x^{-1} t t^{-1}=t t^{-1} x x^{-1}$ and so we also have that $t^{-1}=t^{-1} x x^{-1}$.)

\section{Special involutions}

Let * be an involution on a semigroup $S$. As in [4], we call * a special involution if and only if, for every nonempty finite subset $T$ of $S$,

$$
(\exists t \in T)(\forall u, v \in T) t t^{*}=u v^{*} \Rightarrow u=v .
$$

We have already used (in the proof of 2.2 (iv)) the fact that inversion is a special involution on an inverse semigroup. Other examples of special involutions include word reversal on a free semigroup and hermitian conjugation on the multiplicative semigroup of $n \times n$ complex matrices [4].

An involution on an $F$-algebra $A$ is termed special if and only if it induces a special involution on $(A,$.$) . From 4.1$ we deduce 
TheOREM 5.1. Let $S$ be an inverse semigroup and let $F$ be a subring of $\mathbb{C}$ containing 1 and closed under complex conjugation. Then the natural involution on $F[S]$ is special.

Proof: Let $A$ be a nonempty finite subset of $F[S]$. We show that

$$
(\exists a \in A)(\forall b, c \in A) \quad a a^{*}=b c^{*} \Rightarrow b=c .
$$

Choose $T$ to be a nonempty finite subset of $S$ such that

$$
T \supseteq \bigcup_{b, c \in A} \operatorname{supp}(b-c) \text {. }
$$

By 4.1 , there exists a pseudotrace function $\chi^{T}$ on $F[S]$ relative to $F[T]$. By T4, for all $a \in F[S], \chi^{T}\left(a a^{*}\right) \in F^{+0}$. Hence, since $A$ is finite, we may choose $a \in A$ such that

$$
\chi^{T}\left(a a^{*}\right)=\max \left\{\chi^{T}\left(b b^{*}\right): b \in A\right\} .
$$

Now suppose that $b, c \in A$ are such that $a a^{*}=b c^{*}$. Then $a a^{*}=\left(b c^{*}\right)^{*}=c b^{*}$ and so $(b-c)(b-c)^{*}=b b^{*}+c c^{*}-2 a a^{*}$. Hence, by T1, T4 and the choice of $a$,

$$
0 \leqslant \chi^{T}\left((b-c)(b-c)^{*}\right)=\chi^{T}\left(b b^{*}\right)+\chi^{T}\left(c c^{*}\right)-2 \chi^{T}\left(a a^{*}\right) \leqslant 0,
$$

which shows that $\chi^{T}\left((b-c)(b-c)^{*}\right)=0$. It follows from $T 5^{\prime}$ that $b=c$. Hence ${ }^{*}$ is special.

\section{A CLASS OF COMPLETELY SEMISIMPLE INVERSE SEMIGROUPS}

In this section we use pseudotrace functions (Section 4) to construct a trace function on the real or complex algebra of a particular type of completely semisimple inverse semigroup. The result applies to $\mathbb{C}[S]$ and $\mathbb{R}[S]$, where $S$ is a free inverse semigroup of finite or countably infinite rank.

Lemma 6.1. Let $S$ be an inverse semigroup with semilattice $E$, let $D$ be a $\mathcal{D}$ class of $S$ containing finitely many idempotents and let $F$ be a subring of $\mathbb{C}$ containing 1 and closed under complex conjugation. Then the pseudotrace function $\chi^{D \cap E}$ on $F[S]$ relative to $F[D \cap E]$ satisfies $T 1, T 2, T 3, T 4$ and the additional condition:

T5". $(\forall a \in F[D]) \quad \chi^{D \cap E}\left(a a^{*}\right)=0 \Rightarrow a=0$.

Proof: For simplicity write $\chi:=\chi^{D \cap E}$. By 4.1, $\chi$ satisfies T1, T3 and T4. Note also that

$$
(\forall x \in S) \quad \chi(x)=|\{e \in D \cap E: e x=e\}| \in \mathbb{N} \cup\{0\}
$$


We now show that T2 holds. Let $x, y \in S$. Write

$$
A:=\{e \in D \cap E: e x y=e\}, \quad B:=\{f \in D \cap E: f y x=f\}
$$

Let $e \in A$. Since exy $=e$, we have that $R_{e x}=R_{e}$ and so $e x \in D$. Hence $x^{-1} e x=$ $(e x)^{-1} e x \in D \cap E$. Also, $\left(x^{-1} e x\right) y x=x^{-1} e x$ and so $x^{-1} e x \in B$. Then, as in the proof of 2.3, the mapping $\varphi: A \rightarrow B$ defined by $e \varphi:=x^{-1} e x(e \in A)$ is injective. Thus $|A| \leqslant|B|$. Similarly, we have that $|B| \leqslant|A|$. Hence $\chi(x y)=|A|=|B|=\chi(y x)$. This, together with linearity, establishes T2.

Finally, we show that T5" holds. Let $a \in F[D] \backslash\{0\}$. By 2.2(iv), $a^{*} \theta_{a} \neq 0$ and so there exists $x \in \operatorname{supp}\left(a^{*}\right)$ such that $x \theta_{a} \neq 0$. Hence, by 2.2(iii), $\left(x^{-1} x\right) \theta_{a} \neq 0$. But $\operatorname{supp}(a) \subseteq D$ and so $x \in D$. Consequently, $x^{-1} x \in D \cap E$. Thus

$$
\chi\left(a a^{*}\right)=\sum_{e \in D \cap E}\left(e \theta_{a a^{*}} \mid e\right)=\sum_{e \in D \cap E}\left(e \theta_{a} \mid e \theta_{a}\right) \geqslant\left(\left(x^{-1} x\right) \theta_{a} \mid\left(x^{-1} x\right) \theta_{a}\right)>0
$$

The following result concerns a class of completely semisimple inverse semigroups. (Note that if every $\mathcal{D}$-class of an inverse semigroup $S$ contains finitely many idempotents then $S$ has no bicyclic subsemigroup.)

THEOREM 6.2. Let $S$ be an inverse semigroup such that each $\mathcal{D}$-class contains finitely many idempotents and the set of all $\mathcal{D}$-classes is countably infinite. Let $F=\mathbb{C}$ or $F=\mathbb{R}$. Then $F[S]$ admits a trace function $\chi$ such that, for $x \in S$,

(i) $\chi(x) \in \mathbb{R}$ and $0 \leqslant \chi(x) \leqslant 1$,

(ii) $\chi(x)=1$ if and only if $S$ is a monoid and $x$ is its identity.

ProOf: Let the $\mathcal{D}$-classes of $S$ be denoted by $D_{i}(i \in \mathbb{N})$ and, for each $i \in \mathbb{N}$, define $\chi_{i}: F[S] \rightarrow F$ by writing $\chi_{i}:=\chi^{D_{i} \cap E} /\left|D_{i} \cap E\right|$. Since, by $6.1, \chi^{D_{i} \cap E}$ satisfies conditions $\mathrm{T} 1, \mathrm{~T} 2, \mathrm{~T} 3, \mathrm{~T} 4, \mathrm{~T} 5$ " the same is true for $\chi_{i}$.

Let $i \in \mathbb{N}$. Then, by $6.1 \mathrm{a}$, for all $x \in S$,

$$
\chi_{i}(x)=\left|\left\{e \in D_{i} \cap E: e x=e\right\}\right| /\left|D_{i} \cap E\right| \in \mathbb{Q}
$$

and $0 \leqslant \chi_{i}(x) \leqslant 1$. Thus $0 \leqslant \sum_{i=1}^{\infty} 2^{-i} \chi_{i}(x) \leqslant \sum_{i=1}^{\infty} 2^{-i}=1(x \in S)$. Hence we may define $\chi: S \rightarrow \mathbb{R}$ by the equation $\chi(x):=\sum_{i=1}^{\infty} 2^{-i} \chi_{i}(x)$ and extend it by linearity to $\chi: F[S] \rightarrow F$. Thus, for all $a \in F[S], \chi(a):=\sum_{i=1}^{\infty} 2^{-i} \chi_{i}(a)$. We show that $\chi$ is a trace function on $F[S]$, with the required properties. 
Since each $\chi_{i}$ satisfies $\mathrm{T} 1, \mathrm{~T} 2, \mathrm{~T} 3, \mathrm{~T} 4$ so also does $\chi$. We now show that $\chi$ satisfies T5. First note that, by [2, Theorem 6.45], $\mathcal{D}=\mathcal{J}$ on $S$. Let $a \in F[S] \backslash\{0\}$ and let $D_{k}$ be maximal in the set of $\mathcal{J}$-classes of $S$ that have nonempty intersection with $\operatorname{supp}(a)$. Write $D:=D_{k}$ and $a:=a_{1}+a_{2}$, where $a_{1} \in F[D]$ and $a_{2} \in F[S]$ with $\operatorname{supp}\left(a_{2}\right) \cap D=\emptyset$. Now, for all $x, y \in \operatorname{supp}(a), \chi_{k}\left(x y^{-1}\right)=0$ if either $x \notin D$ or $y \notin D$ : for if $e x y^{-1}=e$ for some $e \in D \cap E$ then, by the maximality of $D, x \in D$ and $y \in D$. Hence $\chi_{k}\left(a a^{*}\right)=\chi_{k}\left(a_{1} a_{1}^{*}\right)$. But $\chi_{k}$ satisfies T5" and $a_{1} \neq 0$ (by the choice of $D)$. Hence $\chi_{k}\left(a a^{*}\right)>0$. Thus, since $\chi_{i}\left(a a^{*}\right) \geqslant 0$ for all $i \in \mathbb{N}$, we have that $\chi\left(a a^{*}\right)>0$. This shows that $\chi$ satisfies T5.

We have already seen that (i) holds for all $x \in S$. Now suppose that $x \in S$ is such that $\chi(x)=1$. Then $\sum_{i=1}^{\infty} 2^{-i}\left(1-\chi_{i}(x)\right)=0$ and so, since $0 \leqslant \chi_{i}(x) \leqslant 1$ for all $i \in \mathbb{N}$, we deduce that $\chi_{i}(x)=1$ for all $i \in \mathbb{N}$. Thus $e x=e$ for all $e \in E$. Hence, for all $y \in S, y x=y\left(y^{-1} y\right) x=y\left(y^{-1} y\right)=y$, which shows that $x$ is a right identity for $S$. But since $S$ has an involution $x$ must be a two-sided identity. Conversely, if $S$ is a monoid with identity $1_{S}$ then clearly $\chi\left(1_{s}\right)=1$. Hence (ii) holds for $x \in S$.

To conclude, we note that a free inverse semigroup $S$ of finite or countably infinite rank satisfies the hypotheses of the theorem [7, Lemma 1.3; 5 , Section 3]. Thus we have

CoROLlaRY 6.3. Let $S$ be a free inverse semigroup of finite or countably infinite rank. Then $\mathbb{C}[S]$ and $\mathbb{R}[S]$ admit trace functions.

In [3] a different method leads to the more general result that if $F$ is a subfield of $\mathbb{C}$ closed under complex conjugation and $S$ is a free inverse semigroup of arbitrary rank then $F[S]$ admits a trace function.

\section{REFERENCES}

[1] B.A. Barnes, 'Representations of the $\ell^{\mathbf{1}}$-algebra of an inverse semigroup', Trans. Amer. Math. Soc. 218 (1976), 361-396.

[2] A.H. Clifford and G.B. Preston, The algebraic theory of semigroups, I and II (Amer. Math. Soc., Providence, 1961 and 1967).

[3] M.J. Crabb and W.D. Munn, 'Trace functions on the algebras of certain $E$-unitary inverse semigroups', Proc. Roy. Soc. Edinburgh Ser. A (to appear).

[4] D. Easdown and W.D. Munn, 'On semigroups with involution', Bull. Austral. Math. Soc. 48 (1993), 93-100.

[5] W.D. Munn, 'Free inverse semigroups', Proc, London Math. Soc. (3) 29 (1974), 385-404.

[6] W.D. Munn, 'A class of contracted inverse semigroup rings', Proc. Roy. Soc. Edinburgh Ser. $A 107$ (1987), 175-196.

[7] N.R. Reilly, 'Free generators in free inverse semigroups', Bull. Austral. Math. Soc. 7 (1972), 407-424. 
School of Mathematics and Statistics University of Sydney

Sydney

New South Wales 2006

Australia
Department of Mathematics

University of Glasgow

Glasgow G12 8QW

Scotland

United Kingdom 